\title{
RIQUEZA E DISTRIBUIÇÃo GEOGRÁFICA DE ESPÉCIES ARBÓREAS DA FAMÍlIA LEGUMINOSAE E IMPLICAÇÕES PARA CONSERVAÇÃo NO CENTRO de Diversidade Vegetal de Cabo Frio, Rio de Janeiro, Brasil ${ }^{1}$
}

\author{
Robson Daumas Ribeiro ${ }^{2,3,4}$ \& Haroldo Cavalcante de Lima $^{3,4}$
}

\begin{abstract}
RESUMo
(Riqueza e distribuição geográfica de espécies arbóreas da família Leguminosae e implicações para conservação no Centro de Diversidade Vegetal de Cabo Frio, Rio de Janeiro, Brasil) Leguminosae apresenta cerca de 727 gêneros e 19.325 espécies distribuídas pelo mundo, sendo uma das principais famílias na composição da flora arbórea de ambientes estacionais. A região de Cabo Frio é o principal núcleo de florestas secas do estado do Rio de Janeiro e por possuir elevada diversidade e endemismo é um dos seis centros de diversidade indicados para a Mata Atlântica. Com o objetivo de conhecer a diversidade de Leguminosae arbóreas no Centro de Diversidade Vegetal de Cabo Frio (CDVCF) e seus padrões de distribuição geográfica foram registrados 81 táxons e reconhecido seis padrões de distribuição. Baseado nos endemismos e em análises de composição de algumas áreas do CDVCF, foi possível indicar os remanescentes de florestas sobre Tabuleiro nas proximidades da Praia da Gorda, Armação dos Búzios e dos morrotes mamelonares dos municípios de Araruama, Iguaba, Saquarema e São Pedro da Aldeia como prioritários para a conservação.
\end{abstract}

Palavras-chave: Fabaceae, Leguminosae, árvore, Mata Atlântica, diversidade e distribuição espacial.

\section{Abstract}

(Species richness and geographic distribution of Leguminosae trees and implications for conservation in the Cabo Frio Center of Plant Diversity, Rio de Janeiro, Brazil) Leguminosae, with about 727 genera and 19,325 species distributed worldwide, is one of the main families in the composition of tree flora of dry environments. The Cabo Frio region holds the main core of dry forests in Rio de Janeiro state. Because of high diversity and endemism, it is one of six Centers of Plant Diversity indicated for the Atlantic Forest. In order to know the diversity of tree Leguminosae of the Cabo Frio Center of Plant Diversity (CDVCF) and their patterns of geographic distribution, 81 taxa were recorded and six distribution patterns were recognized. Based on endemics and composition analyses of several areas of the CDVCF, it was possible to indicate the forest remnants on Tabuleiro in the vicinity of Gorda Beach and on the low hills of Araruama, Iguaba, Saquarema and São Pedro da Aldeia as priorities for conservation.

Key words: Fabaceae, Leguminosae, trees, rain forest, diversity and spatial distribution.

\section{INTRODUÇÃO}

A família Leguminosae possui cerca de 727 gêneros e 19.325 espécies (Lewis et al. 2005), que ocupam os mais variados habitats. O conhecimento de suas espécies, através dos estudos taxonômicos e ecológicos, vem demonstrando a importância desta família na flora tropical, a exemplo de sua significativa riqueza na composição arbórea da Mata Atlântica (Leitão-Filho 1982; Peixoto \& Gentry 1990; Lima \& Guedes-Bruni 1997; Guedes-
Bruni 1998) e de outras formações florestais do Neotrópico (Forero \& Gentry 1988).

A floresta tropical atlântica do Brasil é um importante centro da biodiversidade mundial (Barthlott et al. 1996), que se encontra criticamente ameaçado. Principalmente por encontrar-se em tal situação, este domínio fitogeográfico é considerado prioridade para a conservação (Bibby et al. 1992; Mittermeier et al. 1998; Myers et al. 2000). Para aumentar a eficácia das estratégias de conservação,

Artigo recebido em 05/2008. Aceito para publicação em 02/2009.

${ }^{1}$ Parte da Monografia do primeiro autor, Instituto de Ciências Biológicas e Ambientais da Universidade Santa Úrsula.

${ }^{2}$ Bolsista de Iniciação Cientifica do Conselho Nacional de Desenvolvimento Científico e Tecnológico - PIBIC/CNPq. ${ }^{3}$ Instituto de Pesquisas Jardim Botânico do Rio de Janeiro, R. Pacheco Leão 915, Jardim Botânico, 22460-030, RJ, Brasil.

${ }^{4}$ Autores para correspondência: daumas@jbrj.gov.br; hlima@jbrj.com.br 
particularmente na expectativa de proteger as áreas de grande relevância para a manutenção de ecossistemas naturais, foram definidos seis centros de diversidade vegetal em seu domínio, estando um deles localizado na região dos Lagos, no estado do Rio de Janeiro, o Centro de Diversidade Vegetal de Cabo Frio (CDVCF). A relevante diversidade e endemismo de plantas nesta região (Araujo 1997), bem como a referência como um enclave vegetacional com ligações florísticas do domínio das caatingas (Ab'Saber 1974, 1977), fundamentaram a sua indicação (WWF \& IUCN 1997).

Atualmente, mesmo havendo importantes estudos sobre a diversidade e a composição florística no CDVCF (Araujo 2000; Sá 2006; Sá \& Araujo 2009, neste volume), pouco se sabe sobre as variações destes atributos nas diferentes fitofisionomias. Portanto, obter novos dados é determinante para indicar as prioridades de conservação. Para suprir esta necessidade, os resultados do inventário das Leguminosae arbóreas em diferentes remanescentes florestais do CDVCF são discutidos, com um enfoque na avaliação da riqueza de espécies e nas relações florísticas, bem como para inferir as implicações para a conservação nesta singular região do estado do Rio de Janeiro.

\section{Material e Métodos}

\section{Caracterização da área de estudo}

O Centro de Diversidade Vegetal de Cabo Frio (CDVCF) está localizado na Região dos Lagos, estado do Rio de Janeiro, Brasil, entre as coordenadas $22^{\circ} 30^{\prime}-23^{\circ} 00^{\prime} \mathrm{S}$ e $41^{\circ} 52^{\prime}-42^{\circ} 42^{\prime} \mathrm{W}$ (Fig. 1) e possui cerca de $1.500 \mathrm{~km}^{2}$. É integrado pelos municípios de Araruama, Armação de Búzios, Arraial do Cabo, Cabo Frio, Iguaba, Saquarema e São Pedro da Aldeia, sendo limitada a leste e sul pelo Oceano Atlântico, a oeste pela Serra do Mato Grosso e ao norte

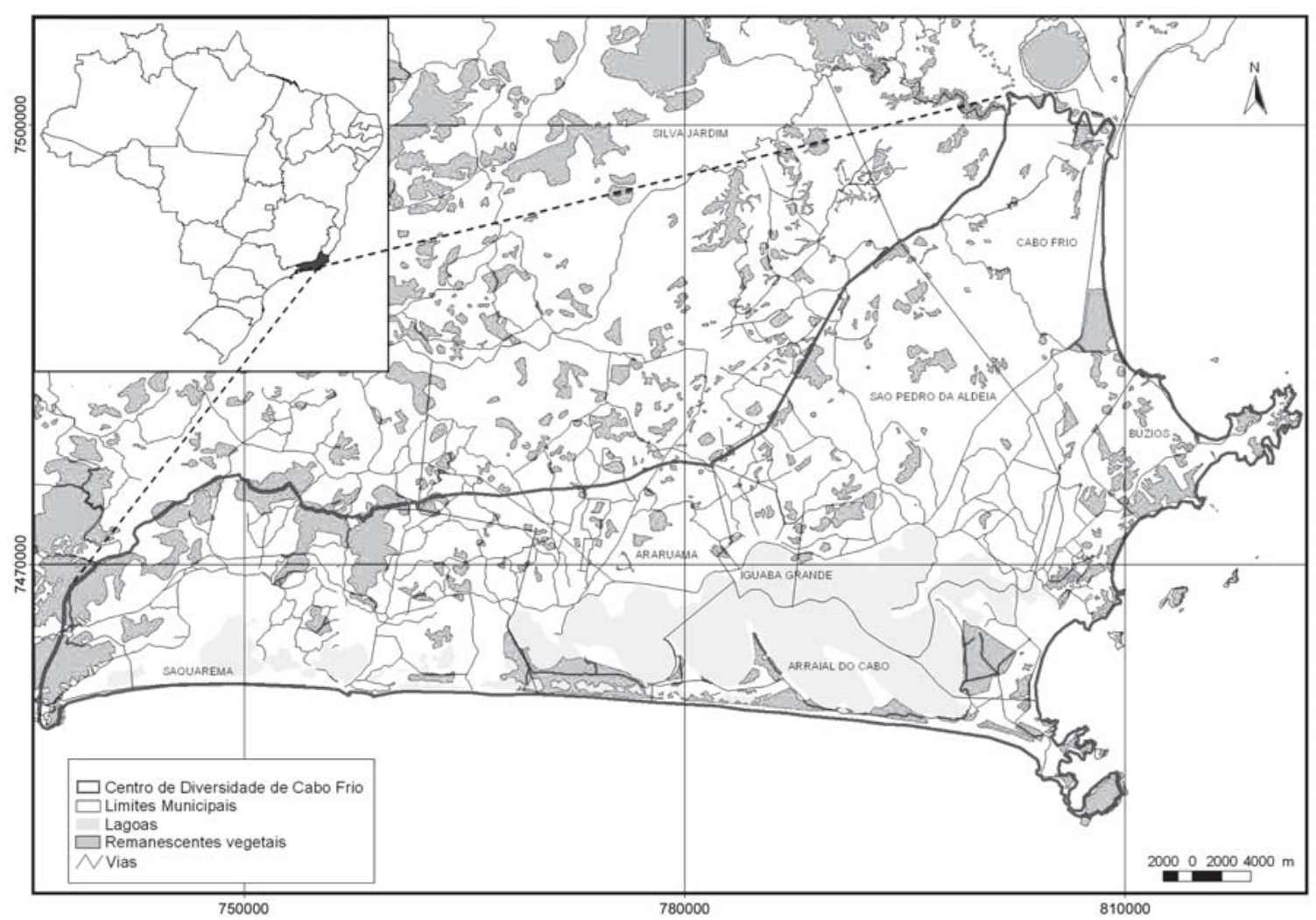

Figura 1 - Localização do Centro de Diversidade Vegetal de Cabo Frio, Rio de Janeiro, Brasil. 
pelos limites superiores da Lagoa de Araruama e pelos cursos inferiores dos rios Una e São João. O clima, de acordo com o sistema de Köppen, é do tipo Bsh, árido quente com temperaturas médias anuais próximas de $25^{\circ} \mathrm{C}$, podendo chegar a $40^{\circ} \mathrm{C}$ no verão. As precipitações pluviométricas ficam em torno de $800 \mathrm{~mm} / \mathrm{ano}$, com cinco meses de seca (WWF \& IUCN 1997). Estas características climatológicas estão mais relacionadas com as áreas entre Cabo Frio e Arraial do Cabo, sendo o restante do CDVCF áreas de transição para o clima tipo Aw, tropical com chuvas de verão e seca no inverno, mas ainda sob forte influência de déficit hídrico (Barbiéri \& Coe Neto 1999).

As fisiografias predominantes na região são as planícies arenosas costeiras, os depósitos alúvios-colúvios, as lagunas, os morros baixos das penínsulas Armação de Búzios e Cabo Frio e as encostas da Serra de Mato Grosso. A altitude varia desde o nível do mar até cerca de 500 metros, sendo menos de $10 \%$ da região acima dos 100 metros (Araujo 2000).

\section{Amostragem e análise dos dados}

O inventário das espécies arbóreas da família Leguminosae no Centro de Diversidade Vegetal de Cabo Frio foi realizado a partir de levantamento bibliográfico (artigos e capítulos de livros, além de teses, dissertações e monografias referentes à região e à família Leguminosae), exame das coleções dos principais herbários do estado do Rio de Janeiro (GUA, R, RB, RBR, RFA e RUSU, aqui citados de acordo com Holmgren et al. 1990) e trabalhos de campo (iniciados em 2002 e finalizados em 2007). Coletas foram realizadas em remanescentes florestais nos sete municípios que integram o CDVCF, utilizando-se o método de coletas assistemáticas através de caminhadas livres.

Adotou-se para árvores a definição de indivíduos lenhosos de tronco não ramificado na base e que alcancem 3 metros ou mais de altura.

As distribuições atuais de espécies foram definidas através dos dados de herbário, bibliografia e trabalhos de campo, enquanto seus respectivos padrões geográficos seguiram modelos disponíveis na literatura (Prance 1979; Mori et al. 1981;
Mori 1990; Pirani 1990; Prado \& Gibbs 1993; Oliveira-Filho \& Ratter 1995; Lima et al. 1997; Lima 2000).

Para o reconhecimento das formações florestais da região do CDVCF foi utilizado o sistema de classificação da vegetação brasileira (Veloso et al. 1991) e sob a denominação genérica de Mata Atlântica são reunidas às formações florestais ombrófilas e estacionais extra-amazônicas, além das formações não florestais associadas, como manguezais, restingas e campos de altitude (Câmara 1991; Joly et al. 1999).

O trabalho de identificação e atualização do material botânico foi realizado com o uso de literatura especializada e revisões taxonômicas recentes, comparação com espécimes do herbário do Instituto de Pesquisas Jardim Botânico do Rio de Janeiro (RB) e consulta a especialistas.

Todas as informações de etiqueta da coleção de Leguminosae arbórea proveniente do CDVCF e depositada no herbário RB estão disponíveis no banco de dados Jabot - Banco de Dados da Flora Brasileira [http://www.jbrj.gov.br/jabot].

\section{Resultados e Discussão}

\section{Diversidade taxonômica}

O resultado do inventário das espécies arbóreas da família Leguminosae no Centro de Diversidade Vegetal de Cabo Frio (CDVCF) é apresentado na Tabela 1, constando da lista de espécies e infra-espécies e os resultados relativos à distribuição geográfica. Foram listados 81 táxons subordinados a 41 gêneros, sendo 19/12 Caesalpinioideae, 23/12 Mimosoideae e 39/17 Papilionoideae.

A diversidade taxonômica de espécies de Leguminosae arbóreas no CDVCF mostrou-se bastante elevada quando comparado com outras áreas estudadas (Tab. 2). A expressiva riqueza de espécies da família Leguminosae na Mata Atlântica foi relatada em vários estudos (Guedes-Bruni et al. 1997; Araujo 2000; Lima 2000; Kurtz \& Araujo 2000; Morim 2006; Morim \& Barroso 2007). O estudo realizado por Lima (2000) para conhecer a variação da riqueza na flora arbórea de Leguminosae em diferentes remanescentes no estado do Rio 
Tabela 1 - Lista das Leguminosae arbóreas registradas para o Centro de Diversidade Vegetal de Cabo Frio com seus respectivos padrões de distribuição geográfica. Abreviações: NEO - Neotropical; ACO - América do Sul Centro-Oriental; SE/NE - Atlântico Sudeste-Nordeste; SE/S - Atlântico Sudeste-Sul; SE - Sudeste; RJ - Rio de Janeiro.

\begin{tabular}{|c|c|}
\hline Táxons & Padrões \\
\hline Abarema cochliacarpos (Gomes) Barneby \& Grimes & SE/NE \\
\hline Acosmium lentiscifolium Spreng. & SE/NE \\
\hline Albizia polycephala (Benth.) Killip & $\mathrm{ACO}$ \\
\hline Amburana cearensis (Allemao) A.C.Sm. & $\mathrm{ACO}$ \\
\hline Anadenanthera colubrina (Vell.) Brenan & $\mathrm{ACO}$ \\
\hline Andira anthelmia (Vell.) Macbr. & $\mathrm{ACO}$ \\
\hline Andira fraxinifolia Benth. & $\mathrm{ACO}$ \\
\hline Andira legalis (Vell.) Toledo & SE \\
\hline Apuleia leiocarpa (Vog.) Macbr. & NEO \\
\hline Barnebydendron riedelii (Tul.) Kirkbride & NEO \\
\hline Bauhinia albicans Vog. & SE/NE \\
\hline Bauhinia forficata Link & $\mathrm{ACO}$ \\
\hline Bauhinia pentandra (Bongard) D. Dietrich & $\mathrm{ACO}$ \\
\hline Caesalpinia echinata Lam. & SE/NE \\
\hline Caesalpinia ferrea Mart. & SE/NE \\
\hline Caesalpinia pluviosa DC. & $\mathrm{ACO}$ \\
\hline Calliandra harrisii Benth. & $\mathrm{ACO}$ \\
\hline Centrolobium tomentosum Benth. & $\mathrm{ACO}$ \\
\hline Chamaecrista ensiformis (Vell.) I. \& B. & ACO \\
\hline Chloroleucon tortum (Mart.) Pittier & RJ \\
\hline Copaifera lucens Dwyer & $\mathrm{SE}$ \\
\hline Copaifera trapezifolia Hayne & $\mathrm{ACO}$ \\
\hline Erythrina speciosa Andr. & $\mathrm{ACO}$ \\
\hline Exostyles venusta Schott & SE/NE \\
\hline Grazielodendron rio-docensis H.C. Lima & $\mathrm{SE}$ \\
\hline Hymenaea courbaril $\mathrm{L}$. & $\mathrm{ACO}$ \\
\hline Inga capitata Desv. & NEO \\
\hline Inga cordistipula Mart. & $\mathrm{SE}$ \\
\hline Inga edulis Mart. & NEO \\
\hline Inga laurina $(\mathrm{Sw}$.$) Willd.$ & NEO \\
\hline Inga marginata Willd. & NEO \\
\hline Inga maritima Benth. & $\mathrm{SE}$ \\
\hline Inga subnuda Salzm. ex Benth. subsp. luschnathiana (Benth.) T.D. Penn. & $\mathrm{SE} / \mathrm{S}$ \\
\hline Lonchocarpus campestris Mart. ex Benth. & SE/NE \\
\hline Lonchocarpus cultratus (Vell.) Az.-Tozzi \& H.C.Lima & NEO \\
\hline Lonchocarpus virgilioides (Vog.) Benth. & SE/NE \\
\hline Machaerium brasiliense Vog. & $\mathrm{ACO}$ \\
\hline Machaerium firmum (Vell.) Benth. & RJ \\
\hline Machaerium fluminense Rudd & SE \\
\hline Machaerium hirtum (Vell.) Stelfeld & NEO \\
\hline Machaerium incorruptibile (Vell.) Benth. & SE/NE \\
\hline Machaerium leucopterum Vog. & $\mathrm{SE} / \mathrm{NE}$ \\
\hline Machaerium nictitans (Vell.) Benth. & $\mathrm{ACO}$ \\
\hline
\end{tabular}




\begin{tabular}{|c|c|}
\hline Táxons & Padrões \\
\hline Machaerium nigrum Vog. & SE \\
\hline Machaerium obovatum Kuhlm. \& Hoehne & $\mathrm{RJ}$ \\
\hline Machaerium pedicelatum Vog. & $\mathrm{SE}$ \\
\hline Machaerium punctatum (Poir.) Pers. & $\mathrm{ACO}$ \\
\hline Machaerium stipitatum (DC.) Vog. & ACO \\
\hline Melanoxylon brauna Schott & ACO \\
\hline Mimosa arenosa (Willd.) Poiret var. arenosa & NEO \\
\hline Mimosa bimucronata (DC.) Kuntz. & NEO \\
\hline Myrocarpus fastigiatus Allemao & SE/NE \\
\hline Myrocarpus frondosus Allemao & $\mathrm{ACO}$ \\
\hline Ormosia arborea (Vell.) Harms & $\mathrm{ACO}$ \\
\hline Parapiptadenia pterosperma (Benth.) Brenan & SE/NE \\
\hline Peltogyne discolor Vog. & $\mathrm{SE} / \mathrm{NE}$ \\
\hline Peltophorum dubium (Spreng.) Taub. & NEO \\
\hline Piptadenia gonoacantha (Mart.) Macbr. & $\mathrm{ACO}$ \\
\hline Piptadenia paniculata Benth. & ACO \\
\hline Plathymenia reticulata Benth. & ACO \\
\hline Platymiscium floribundum Vog. var. floribundum & ACO \\
\hline Platymiscium floribundum Vog. var. latifolium (Benth.) Benth. & $\mathrm{SE} / \mathrm{S}$ \\
\hline Platymiscium floribundum Vog. var. nitens (Vog.) Klitgaard & $\mathrm{ACO}$ \\
\hline Poecilanthe falcata (Vell.) Heringer & SE/NE \\
\hline Pseudopiptadenia contorta (DC.) G.P. Lewis \& M.P. Lima & $\mathrm{ACO}$ \\
\hline Pseudopiptadenia inaequalis (Benth.) Rauchert & SE \\
\hline Pseudopiptadenia schumanniana (Taub.) G.P. Lewis \& M.P. Lima & RJ \\
\hline Pterocarpus rohrii Vahl & NEO \\
\hline Pterogyne nitens Tul. & $\mathrm{ACO}$ \\
\hline Senegalia bahiensis (Benth.) Seigler \& Ebinger & SE/NE \\
\hline Senegalia polyphylla (DC.) Britton \& Rose & NEO \\
\hline Senna macranthera (Collad.) I. \& B. & NEO \\
\hline Senna multijuga (L.C. Rich.) I. \& B. var. lindleyana (Gardn.) I. \& B. & ACO \\
\hline Senna silvestris (Vell.) I. \& B. var. silvestris & NEO \\
\hline Swartzia apetala Raddi var. apetala & SE/NE \\
\hline Swartzia apetala Raddi var. glabra (Vog.) Cowan & SE/NE \\
\hline Swartzia flaemingii Raddi var. flaemingii & SE/NE \\
\hline Swartzia glazioviana (Taub.) Glaziou & RJ \\
\hline Swartzia myrtifolia J.E. Smith var. elegans (Schott) Cowan & $\mathrm{ACO}$ \\
\hline Sweetia fruticosa Spreng. & ACO \\
\hline Zollernia glabra (Spreng.) Yakovl. & SE \\
\hline
\end{tabular}

de Janeiro indicou uma maior diversidade em altitudes abaixo de 500 metros, principalmente nos maciços litorâneos isolados da cadeia central da Serra do Mar. A elevada riqueza de árvores da família Leguminosae no CDVCF corrobora com estes dados, já que a referida região está enquadrada nesses limites altitudinais.
É interessante ressaltar que a riqueza de gêneros entre as áreas estudadas mostrou-se mais alta em locais com floresta estacional (Caratinga, CDVCF), contrastando com a menor riqueza em locais com floresta ombrófila (Cairuçu, Itatiaia e Macaé de Cima). Este resultado parece seguir uma tendência geral observada para a família, cujas áreas de maior 
Tabela 2 - Quantificação de gêneros e espécies de Leguminosae arbóreas em outras áreas da região Sudeste do Brasil. Abreviações: CDVCF, Centro de Diversidade Vegetal de Cabo Frio-RJ (presente estudo); CAIRUÇU, APA de Cairuçu-RJ (Marques 1997); CARATINGA, Estação Biológica de Caratinga-MG (Mendonça Filho 1996); ITATIAIA, Parque Nacional de Itatiaia (Morim 2002); MACAÉ DE CIMA, Reserva Ecológica de Macaé de Cima-RJ (Lima et al. 1994).

\begin{tabular}{lccccc}
\hline & CDVCF & CAIRUÇU & CARATINGA & ITATIAIA & $\begin{array}{c}\text { MACAÉ } \\
\text { DE CIMA }\end{array}$ \\
\hline $\mathrm{N}^{\text {o de gêneros }}$ & 41 & 20 & 41 & 30 & 18 \\
$\mathrm{~N}^{\text {o de espécies }}$ & 81 & 36 & 65 & 47 & 35 \\
\hline
\end{tabular}

diversificação estão localizadas em ambientes estacionais (Lewis et al. 2005). Tal diversificação supõe-se muito antiga, pois remontaria ao Terciário, quando as florestas secas dominavam as principais regiões do mundo (Pennington et al. 2004). Além disso, a associação da família com bactérias fixadoras de nitrogênio tem sido apontada como um meio eficiente para a ocupação de ambientes pobres em nutrientes e em regeneração (Franco et al. 1992; Mckey 1994; Sprent 1994; Campello 1997; Faria 1997; Faria \& Lima 2002; Faria et al. 2006). Portanto, entre as possíveis explicações para a elevada riqueza de leguminosas arbóreas no CDVCF, uma das mais consistentes pode estar relacionada com a elevada diversidade desta família em florestas estacionais tropicais e a alta capacidade desta família em ocupar locais com solos pobres em nutrientes e áreas degradadas, que são freqüentes na paisagem do CDVCF e de toda a Mata Atlântica.

Os gêneros e os respectivos números de espécies/infra-espécies estão apresentados na Tabela 3. Três gêneros estão representados por cinco ou mais espécies, enquanto 12 possuem duas ou três espécies e os 26 gêneros restantes por apenas uma espécie. Destaca-se o gênero Machaerium com 12 espécies, cuja elevada riqueza já foi constatada para a região neotropical (Hoehne 1941; Rudd 1987; Mendonça-Filho 2002), principalmente em formações vegetais submetidas à baixa pluviosidade (Lima 2000). Isto reforça a suposição sobre a contribuição de elementos relacionados com florestas estacionais para a composição florística do CDVCF. Por outro lado, a riqueza de espécies em Inga e Swartzia, respectivamente sete e cinco espécies, gêneros com preferência por ambientes úmidos (Cowan 1967; Pennington 1997; Mansano 1997; Richardson et al. 2001) pode também indicar a influência das florestas ombrófilas adjacentes, em particular da cadeia da Serra do Mar, na composição de Leguminosae nas florestas do CDVCF. Estes resultados sugerem que a elevada riqueza florística constatada para esta região (Araujo et al. 1998; Araujo 2000; Sá 2006; Sá \& Araujo, 2009, neste volume) pode também ser explicada por essa co-ocorrência de espécies.

A análise do inventário de Leguminosae arbóreas no CDVCF também contribui para um melhor conhecimento da diversidade florística da Mata Atlântica. Foram registrados cinco táxons citados pela primeira vez para o estado do Rio de Janeiro, sendo eles Amburana cearensis, Lonchocarpus campestris, Machaerium fluminense, M. nigrum e Senegalia bahiensis. Estas novas descobertas de leguminosas arbóreas nos remanescentes de Mata Atlântica deste estado, onde estudos florísticos vêm sendo realizados desde o século XVIII (Lima 1995), demonstram o quão distante estamos de um inventário completo da diversidade destas florestas. Este resultado é surpreendente, pois ressalta a insuficiência de amostragem de coleta em alguns trechos deste bioma no estado do Rio de Janeiro e a necessidade de estudos 
Tabela 3 - Quantificação do número de espécies e infra-espécies por gêneros no CDVCF.

\begin{tabular}{llll}
\hline Machaerium & 12 & Barnebydendron & 1 \\
Inga & 7 & Calliandra & 1 \\
Swartzia & 5 & Centrolobium & 1 \\
Andira & 3 & Chamaecrista & 1 \\
Bauhinia & 3 & Chloroleucon & 1 \\
Caesalpinia & 3 & Erythrina & 1 \\
Lonchocarpus & 3 & Exostyles & 1 \\
Platymiscium & 3 & Grazielodendron & 1 \\
Pseudopiptadenia & 3 & Hymenaea & 1 \\
Senna & 3 & Melanoxylon & 1 \\
Copaifera & 2 & Ormosia & 1 \\
Mimosa & 2 & Parapiptadenia & 1 \\
Myrocarpus & 2 & Peltogyne & 1 \\
Piptadenia & 2 & Peltophorum & 1 \\
Senegalia & 2 & Platymenia & 1 \\
Abarema & 1 & Poecilanthe & 1 \\
Acosmium & 1 & Pterocarpus & 1 \\
Amburana & 1 & Pterogyne & 1 \\
Albizia & 1 & Sweetia & 1 \\
Anadenanthera & 1 & Zollernia & 1 \\
Apuleia & 1 & TOTAL & $\mathbf{4 1 / 8 1}$ \\
\hline
\end{tabular}

taxonômicos em gêneros arbóreos. Em termos de avaliação da diversidade, o resultado alcançado para o CDVCF é muito relevante, pois indica que a diversidade florística de florestas estacionais fluminenses pode estar subestimada, contrastando com o conhecimento atual das florestas ombrófilas, como já foi apontado por Janzen (1997) e Mooney et al. (1995) para outras regiões tropicais.

No estado do Rio de Janeiro, poucos são os estudos florísticos em florestas estacionalmente secas (Farág 1999; Silva \& Nascimento 2001; Spolidoro 2001; Sá 2006; Nascimento \& Lima 2008; Maioli-Azevedo 2008). No CDVCF a totalidade dos trabalhos realizados se refere às florestas sobre áreas de colinas, vestígios de tabuleiros da formação Barreiras e planícies arenosas. Assim, são ainda precárias as informações florísticas sobre as florestas das elevações mais úmidas (Sá 2006), entre as quais se destacam as serras da Castelhana e de Mato Grosso. Futuros inventários nestas serras provavelmente darão conhecimento a novos registros botânicos, corroborando para fortalecer a indicação da região de Cabo Frio como um centro de diversidade.

\section{Padrões de distribuição geográfica}

Foram definidos os padrões de distribuição geográfica de 81 táxons específicos ou infraespecíficos (Tab. 1). Os padrões de distribuição estão delineados na Figura 2 e sumarizados na Tabela 4.

\section{Neotropical (NEO)}

Representado por 15 espécies (ca. 19\%) ocorrentes no CDVCF, que abrange a América do Sul e Central e estende-se até o México. Destacam-se neste padrão as espécies generalistas que ocorrem nas mais distintas formações vegetais, como também verificados por Lima (2000) e Morim (2006). Pterocarpus rohrii (Fig. 3a) é uma espécie que habita frequentemente as florestas ombrófilas e estacionais neotropicais, mas também se estende pelas matas ciliares até às áreas de cerrado e caatinga. Algumas espécies, como por exemplo, Inga capitata, I. edulis e I. laurina, possuem ampla distribuição associada às principais bacias hidrográficas, ocorrendo preferencialmente em florestas ribeirinhas e planícies de inundação (Pennington 1997). Apenas Barnebydendron riedelii mostra uma distribuição disjunta, ocorrendo em florestas da América Central, da Amazônia e do Brasil Sudeste (Warwick et al. 2008).

\section{América Centro-Oriental (ACO)}

Representado por 31 espécies (ca. 38\%) ocorrentes no CDVCF, que abrange o Brasil Central, Nordeste e Sudeste, podendo se estender até o Nordeste da Bolívia, Paraguai, Uruguai e Argentina. Categorizadas sob este padrão, a maioria das espécies do CDVCF mostrou a distribuição associada preferencialmente com as áreas de florestas estacionais tropicais, a exemplo de Amburana cearensis (Fig. 3b), Anadenanthera colubrina, Caesalpinia pluviosa e Machaerium brasiliense (Oliveira-Filho \& Ratter 1995; Lewis 1987). Entretanto, foi ainda observado que algumas espécies possuem uma distribuição 


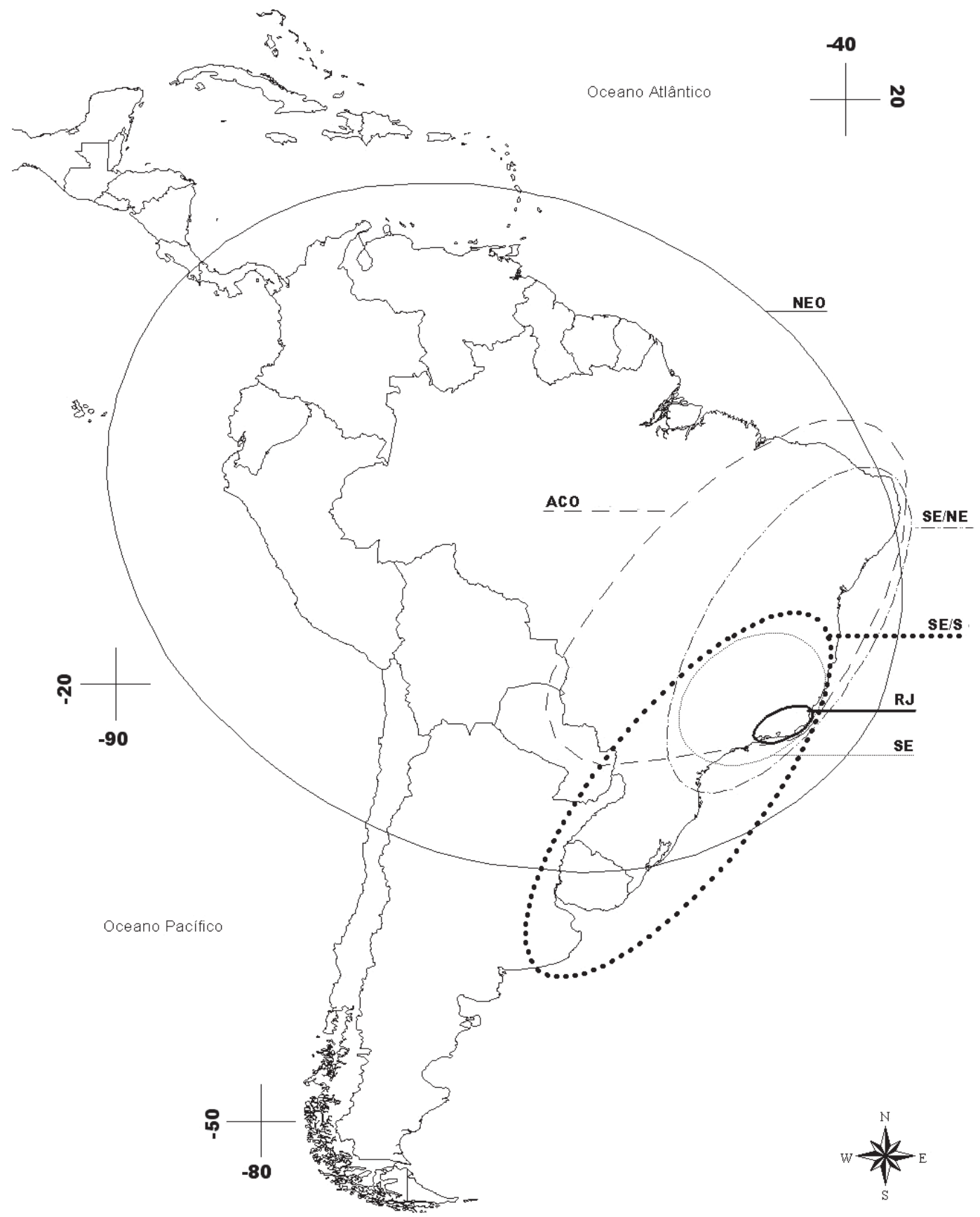

500

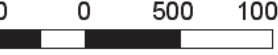

Figura 2 - Delimitação geográfica dos padrões de distribuição (abreviados de acordo com a Tabela 1) verificados para as Leguminosae arbóreas do Centro de Diversidade Vegetal de Cabo Frio. 
Tabela 4 - Número de espécies e porcentagem dos padrões de distribuição geográfica. Abreviação dos Padrões: NEO - América do Sul, Central e México; ACO - Centro-Oriental, Nordeste da Bolívia, Paraguai, Uruguai e Argentina; SE/NE - Costa atlântica, desde o estado de São Paulo até o Ceará; SE/S - Costa atlântica, desde o estado do Espírito Santo até a Argentina; SE - estados do Rio de Janeiro, Espírito Santo, Minas Gerais e São Paulo; RJ - Endêmicas do estado do Rio de Janeiro.

\begin{tabular}{lcl}
\hline Padrões & Node Espécies $^{\mathbf{0}}$ & $\mathbf{\%}$ \\
\hline $\mathrm{NEO}$ & 15 & 19 \\
$\mathrm{ACO}$ & 31 & 38 \\
$\mathrm{SE} / \mathrm{NE}$ & 18 & 22 \\
$\mathrm{SE}$ & 10 & 12 \\
$\mathrm{RJ}$ & 5 & 6 \\
$\mathrm{SE} / \mathrm{S}$ & 2 & 2 \\
\hline
\end{tabular}

associada às florestas ombrófilas, por exemplo, Ormosia arborea, Pseudopiptadenia contorta e Swartzia myrtifolia var. elegans. Estas espécies alcançam o Brasil Central e Nordeste, onde ocorrem em redutos florestais, em geral ribeirinhos ou montanos (Lima 2000).

\section{Atlântico Sudeste-Nordeste (SE/NE)}

Representado por 18 espécies (ca. 22\%) ocorrentes no CDVCF, que abrange a extensão Sudeste-Nordeste da costa atlântica brasileira. A maioria das espécies com este limite de distribuição mostrou também preferência por florestas estacionais, algumas exclusivas das florestas litorâneas e restingas, como por exemplo, Caesalpinia echinata (Fig. 3c) e Lonchocarpus virgilioides. Essas espécies, dentre outras, possuem uma distribuição associada às florestas estacionais sobre tabuleiros de formação barreiras e planícies arenosas de origem marinha. Entretanto, foi verificado ainda que algumas espécies como Bauhinia albicans e Machaerium leucopterum possuem uma distribuição disjunta, ocorrendo em remanescentes florestais do CDVCF e da caatinga, no nordeste do Brasil (Lima 2000).

\section{Atlântico Sudeste-Sul (SE/S)}

Representado por duas espécies (ca. 2\%) ocorrentes no CDVCF, que abrange a extensão Sudeste-Sul da costa atlântica, desde o estado do Espírito Santo no território brasileiro até a Argentina. Estas espécies, Inga subnuda subsp. luschnathiana e Platymiscium floribundum var. latifolium (Fig. 3d), ocorrem preferencialmente em florestas ombrófilas ou florestas ribeirinhas (Pennington 1997; Klitgaard 2005).

\section{Sudeste (SE)}

Representado por 10 espécies (ca. 12\%) ocorrentes no CDVCF, que abrange a porção central da costa atlântica brasileira. A maioria das espécies têm preferência pelas florestas estacionais de terras baixas, como por exemplo, Grazielodendron rio-docensis (Fig. 3e) e Machaerium fluminense (Lima 1983; Ribeiro \& Lima 2007). Entretanto, foi constatado que algumas espécies também ocorrem em restinga, a exemplo de Andira legalis e Inga maritima. Neste padrão, Pseudopiptadenia inaequalis é a única espécie com preferência por florestas ombrófilas.

\section{Rio de Janeiro (RJ)}

Representado por cinco espécies (ca. $6 \%$ ) ocorrentes no CDVCF, e que se caracteriza pelas espécies endêmicas do estado do Rio de Janeiro. Incluído neste padrão estão às espécies Machaerium obovatum e Swartzia glazioviana (Mendonça Filho 2002; Mansano \& AzevedoTozzi 1999), que possuem distribuição restrita aos limites do CDVCF e as espécies Chloroleucon tortum (Fig. 3f), Machaerium firmum e Pseudopiptadenia schumanniana que possuem uma distribuição mais abrangente dentro do estado (Lima 2000; Ribeiro \& Lima 2007).

Estudos biogeográficos sobre plantas arbóreas das formações florestais do Brasil Oriental Atlântico podem ser influenciados pelo esforço de coleta concentrado em certas regiões, como também pelas lacunas de conhecimento botânico em algumas áreas (Mori et al. 1981; Mansano \& Azevedo-Tozzi 1999). Entretanto, no 

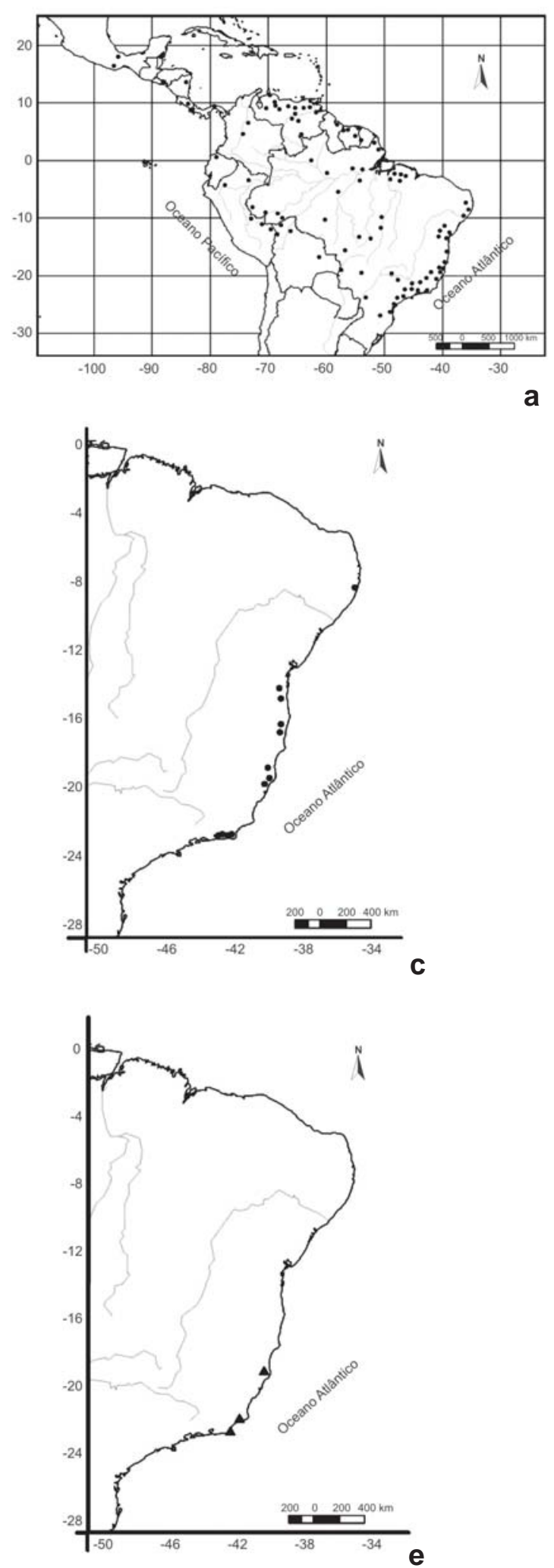
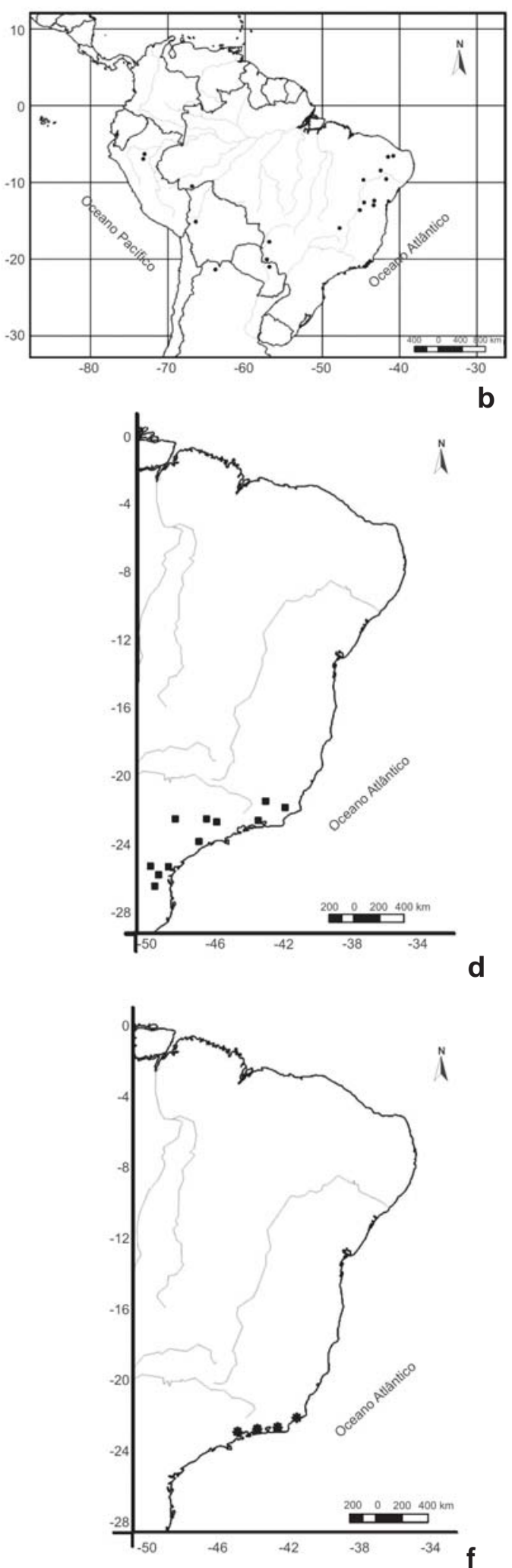

Figura 3 - Distribuição geografica de Leguminosae arbóreas: a. Pterocarpus rohrii; b. Amburana cearensis; c. Caesalpinia echinata; d. Platymiscium floribundum var. latifolium; e. Grazielodendron rio-docensis; f. Chloroleucon tortum. 
caso da família Leguminosae, as coletas extensivas realizadas nos últimos anos já permitem traçar com razoável precisão, pelo menos no Brasil Sudeste, os limites de distribuição da maioria das espécies arbóreas. Neste contexto, os resultados alcançados no presente estudo, com as espécies arbóreas de Leguminosae do CDVCF, corroboram para que este grupo de plantas seja indicado como modelo para facilitar a compreensão dos endemismos e relacionamentos florísticos das formações florestais no Neotrópico.

A vegetação de aspecto xeromórfico do CDVCF, particularmente nos maciços costeiros dos municípios de Armação de Búzios, Arraial do Cabo e Cabo Frio, tem sido interpretada como uma área disjunta da caatinga nordestina (Ururahy et al. 1983). Entretanto, outras avaliações têm proposto que uma zona de florestas estacionais penetra na porção norte do estado do Rio de Janeiro (Oliveira-Filho \& Fontes 2000), e mais recentemente, que essa formação florestal se estende até a região dos Lagos (Lima 2000; Nascimento \& Lima 2008).

$\mathrm{Na}$ presente análise constatou-se o elevado percentual dos padrões NEO e ACO, respectivamente $19 \%$ e $38 \%$ (Tab. 4). Nestes padrões predomina espécies com limites de distribuição associados a ambientes de baixa pluviosidade (em geral abaixo de $1600 \mathrm{~mm}$ anuais e 5-6 meses com menos de $100 \mathrm{~mm}$ ). Prado (1991) e Prado \& Gibbs (1993) sustentam que a flora de áreas secas na América do Sul pode representar um relicto de uma floresta estacional amplamente distribuída nas fases mais secas do Pleistoceno. Atualmente já existem evidências de uma origem mais antiga, apoiada por registros fósseis datados do Mioceno/Plioceno (Pennington et al. 2006). Com base neste modelo, os padrões de ampla distribuição em espécies arbóreas de Leguminosae sustentam a forte conexão florística entre a vegetação atual do CDVCF $\mathrm{e}$ as florestas estacionais neotropicais.

Por outro lado, o percentual de espécies com padrões mais restritos, respectivamente SE/NE (22\%), SE/S (2\%), SE (12\%) e RJ $(6 \%)$, indica a presença de um componente endêmico à porção oriental atlântica da América do Sul (Mata Atlântica sensu lato). A análise da preferência de habitat das espécies com estes padrões mostrou um predomínio por formações litorâneas (florestas e restingas), sugerindo ainda a diferenciação florística entre florestas estacionais litorâneas e interioranas. Tal constatação sustenta que elementos florísticos restritos a florestas estacionais litorâneas são relevantes na vegetação do CDVCF.

As relações entre a vegetação do CDVCF e a caatinga nordestina foram pouco consistentes com os padrões de distribuição de leguminosas arbóreas aqui encontrados. São poucas as espécies que ocorrem na caatinga e se estendem até as florestas do Brasil Sudeste. Entretanto, como notado por Prado \& Gibbs (1993)e Queiroz (2006), usualmente espécies com este padrão ocorrem em formações florestais no domínio da caatinga e são associadas às florestas estacionais tropicais. Sá (2006) também constatou alta dissimilaridade entre a composição florística da vegetação arbórea do CDVCF e as áreas de caatinga do semiárido brasileiro.

As espécies com preferência por florestas ombrófilas também são pouco representadas no CDVCF. Plantas com padrão NEO, como Inga edulis, I. laurina e Lonchocarpus cultratus, possuem uma distribuição geralmente associada às florestas ribeirinhas. Entre os representantes dos padrões SE e SE/S, espécies com esta preferência de habitat em geral ocorrem em redutos de maior altitude do CDVCF, tais como a Serra de Mato Grosso e da Castelhana.

\section{Implicações para conservação}

Em uma análise geral, grande parte da região do CDVCF está inserida em Unidades de Conservação, principalmente em categorias de uso sustentável, tais como Área de Proteção Ambiental (APA) e Reserva Extrativista (RESEX). No entanto, face às dificuldades de implantação e gestão destes modelos de unidades, os impactos antrópicos não estão sendo contidos efetivamente, nem assegurada à proteção dos remanescentes florestais mais representativos. 
Nas áreas com vegetação natural, particularmente nos fragmentos florestais, foi registrada uma grande riqueza de espécies arbóreas da família Leguminosae, incluindo 11 espécies de interesse conservacionista devido ao grau de endemismo em florestas de terras baixas ou por constarem na lista de espécies ameaçadas da flora brasileira (Tab. 5).

Machaerium obovatum e Swartzia glazioviana são as duas únicas espécies endêmicas ao CDVCF. Até o momento foram registradas nas matas baixas e secas sobre os costões e colinas litorâneas, ou em pequenos redutos de restinga na área de entorno destas formações. Além dessas espécies, Andira legalis, Chloroleucon tortum, Grazielodendron riodocensis e Inga maritima devido à distribuição restrita ao estado do Rio de Janeiro ou ao Sudeste brasileiro, também apóiam a alta relevância dos remanescentes destas formações para conservação da diversidade biológica no CDVCF. Destacase ainda que nesta singular fito-fisionomia foram registradas algumas populações de pau-brasil (Caesalpinia echinata), espécie ameaçada de extinção que pode servir de suporte para a efetivação de medidas de proteção dos remanescentes florestais.

A região interiorana do $\mathrm{CDVCF}$, mesmo estando quase completamente devastada e ocupada por pastos, ainda possui importantes remanescentes de florestas estacionais sobre seus morrotes mamelonares. Estes remanescentes possuem alta riqueza de Leguminosae arbóreas, onde foram observadas as principais populações de espécies exploradas no passado devido ao seu potencial madeireiro, tais como a braúna (Melanoxylon brauna), a cerejeira (Amburana cearensis), o pau-brasil (Caesalpinia echinata) e o vinhático (Plathymenia reticulata).

Os resultados encontrados na análise de diversidade e endemismos em espécies arbóreas de Leguminosae reforçam a necessidade de conservação da vegetação remanescente no CDVCF. Entre os trechos mais relevantes, destacam-se os fragmentos florestais nas proximidades da Praia da Gorda no município de Armação de Búzios e outros localizados na região mais afastadas do litoral, nos municípios de Araruama, Iguaba, Saquarema e São Pedro da Aldeia, que são indicados como prioritários para implementação de uma política efetiva para proteção.

\section{Conclusões}

A elevada riqueza de espécies arbóreas da família Leguminosae constatada no presente estudo para o CDVCF corrobora a indicação de que a Região Sudeste do Brasil constitui num importante centro de diversidade.

A discussão elaborada para explicar esta riqueza de espécies da família Leguminosae

Tabela 5 - Espécies arbóreas da família Leguminosae de interesse conservacionista no CDVCF. * incluídas na Lista Oficial das Espécies da Flora Brasileira Ameaçadas de Extinção (BRASIL 2008) e categorizadas de acordo com avaliação no workshop (BIODIVERSITAS 2005).

\begin{tabular}{ll}
\hline Espécies & Categorias \\
\hline Andira legalis (Vell.) Toledo & Endêmica SE \\
Amburana cearensis (Allemão) A.C.Sm.* & Vulnerável(VU) \\
Caesalpinia echinata L.* & Em perigo(EN) \\
Chloroleucon tortum (Mart.) Pittier & Endêmica RJ \\
Grazielodendron riodocensis H.C. Lima* & Vulnerável(VU) \\
Inga maritima Benth. & Endêmica SE \\
Machaerium firmum (Vell.) Benth. & Endêmica RJ \\
Machaerium fluminense Rudd & Endêmica SE \\
Machaerium obovatum Kuhlm. \& Hoehne* & Vulnerável(VU) \\
Melanoxylon brauna Schott* & Vulnerável(VU) \\
Swartzia glazioviana (Taub.) Glaziou* & Em perigo(EN) \\
\hline
\end{tabular}


arbóreas no CDVCF não pode ser empregada inteiramente para outras famílias de plantas, já que a capacidade de fixação de nitrogênio e uma maior diversificação desta família em ambientes estacionais sejam características peculiares. Entretanto, padrão de diversidade semelhante também foi verificado para outros grupos de plantas.

A maioria das espécies de Leguminosae arbóreas inventariadas no CDVCF possui ampla distribuição e constitui elementos floristicos relictuais de uma antiga floresta estacional com extensões pelo Neotropico. Tal observação está relacionada ao padrão de distribuição dessas espécies atualmente associado às florestas adjacentes a Diagonal Seca (corredor vegetacional que liga os domínios da Caatinga, Cerradoe Chaco).

Os resultados apóiam a necessidade de uma política efetiva de proteção para os remanescentes florestais no CDVCF, sendo indicados como prioritários os fragmentos litorâneos no entorno da Praia da Gorda e na região mais afastada do litoral, nos municípios de Araruama, Iguaba, Saquarema e São Pedro da Aldeia.

\section{Agradecimentos}

Ao Conselho Nacional de Desenvolvimento Científico e Tecnológico (CNPq) pela bolsa de iniciação científica (PIBIC) concedida ao primeiro autor; a Petrobras e ao Programa Mata Atlântica/JBRJ convênio 610.4.025.02.3 pelo apoio nos trabalhos de campo; à Fauna \& Flora International (FFI) pelo apoio aos inventários em áreas remanescentes de pau-brasil; a Cyl Farney Catarino de Sá, Heloisa G. Dantas e Daniela Fernandes pela ajuda no campo, incentivo e informações concedidas sobre o Centro de Diversidade Vegetal de Cabo Frio; a Regina H. P. Andreata pelo apoio; a Clarisse P. Farias e José E. C. Meireles pela elaboração das figuras e a Veronica Maioli-Azevedo pela ajuda na elaboração do abstract.

\section{REFERÊNCIAS BIBLIOGRÁFICAS}

Ab'Saber, A. N. 1974. O domínio morfoclimático semi-árido das caatingas brasileiras. Geomorfologia 43: 1-39.
Ab'Saber, A.N. 1977. Espaços ocupados pela expansão dos climas secos na América do Sul por ocasião dos períodos glaciais quaternários. Paleoclimas 3: 1-19.

Araujo, D. S. D. 1997. The Cabo Frio region. In: Davis, S. D.; Heywood,V. H.; HerreraMcBryde, O.; Villa-Lobos, J. \& Hamilton, A. C. (eds.). Centres of Plant Diversity: a guide and strategy for their conservation. Vol 3: The Americas. WWF/IUCN. Oxford. Pp. 373-375.

.2000. Análise florística e fitogeográfica das restingas do estado do Rio de Janeiro. Tese de Doutorado. Universidade Federal do Rio de Janeiro, Rio de Janeiro, 169p.

; Lima, H.C.; Farág, P.R.C.; Lobão, A.Q.; Sá, C.F.C. \& Kurtz, B.C. 1998. O centro de diversidade vegetal de Cabo Frio: levantamento preliminar da flora. Anais do IV Simpósio de Ecossistemas Brasileiros Vol. 3. Publicação ACIESP 104: 147-157.

Barbiéri, E. \& Coe Neto, R. 1999. Spatial and temporal variation of rainfall of the east fluminense coast and atlantic serra do mar, State of Rio de Janeiro, Brazil. In: Knoopers, B.; Bidone, E. D. \& Abrão, J. J. (eds.). Environmetal Geochemistry of Coastal Lagoon Systems. Série Geoquímica Ambiental 6: 47-56.

Barthlott, W., Lauer, W. \& Placke, A. 1996. Global distribution of species diversity in vascular plants: towards a world map of phytodiversity. Erdkunde 50(4): 317-327.

Bibby, C. J.; Collar, N. J.; Crosby, M. J.; Heath, M. F.; Imboden, C.; Johnson, T. H.; Long, A. J.; Statterfield, A. J. \& Thirgood, S. J. 1992. Putting biodiversity on the map: priority areas for global conservation. International Council for Bird Conservation, Cambridge, 96p.

BIODIVERSITAS. 2005. Lista da flora brasileira ameaçada de extinção segundo avaliação no workshop: http://www. biodiversitas.org.br/florabr/lista_florabr.pdf.

BRASIL. 2008. Instrução Normativa $n^{\circ} 6$, de 23 de setembro de 2008. Anexo 1. Lista oficial das espécies da flora brasileira 
ameaçada de extinção. Brasília, Ministério do Meio Ambiente, 55p.

Câmara, I. G. 1991. Plano de ação para a Mata Atlântica. Fundação SOS Mata Atlântica/ Ed. Interação, São Paulo.

Campello, E. F. C. 1997. O papel das leguminosas arbóreas noduladas e micorrizas na recuperação de áreas degradadas. In: Curso de Atualização em Recuperação de áreas degradadas. FUPEF. Pp. 9-16.

Cowan, R. S. 1967. Swartzia (Leguminosae, Caesalpinioideae, Swartzieae). Flora Neotropica Monografia 1: 1-228.

Farág, P. R. C. 1999. Estrutura do estrato arbóreo de mata litorânea semicaducifólia sobre solo arenoso no município de Búzios, RJ.Dissertação de Mestrado. Universidade Federal do Rio de Janeiro, Rio de Janeiro, 92p.

Faria, S. M. 1997. Recuperação de áreas degradadas. In: Reis, V. L. (ed.). Recursos hídricos no Tocantins. CNPq/UNITINS. Pp. 54-57.

\& Lima, H.C. 2002. Levantamento de nodulação em leguminosas arbóreas e arbustivas em áreas de influência da Mineração Rio do Norte-Porto Trombetas/ PA. Série Documentos No 159. Embrapa Agrobiologia, Seropédica, RJ. Pp. 1-32.

; Lima, H.C.; Ribeiro, R.D.; Castilho, F.C. \& Henriques, J.C. 2006. Nodulação em espécies leguminosas da região de Porto Trombetas, Oriximiná, estado do Pará e seu potencial uso no reflorestamento de bacias de rejeito do lavado de bauxita. Série Documentos $N^{\circ}$ 209. Embrapa Agrobiologia, Seropédica, RJ. Pp. 1-24.

Forero, E \& Gentry, A. H. 1988. Neotropical plant distribuition patterns with emphasis on northwestern South America: a preliminary overview. In: Vanzolini, P. E. \& Heyer, W. R. (eds.). Proceedings of a Workshop on Neotropical distribuition patterns. Academia Brasileira de Ciências, Rio de Janeiro. Pp. 21-37.

Franco, A. A.; Campello, E. F.; Silva, E. M. R. \& Faria, S. M. 1992. Revegetação de solos degradados. Comunicado Técnico $N^{\circ}$ 09. Embrapa Agrobiologia, Seropédica, RJ. Pp. 1-09.

Guedes-Bruni, R. R. 1998. Composição, estrutura e similaridade florística de dossel em seis unidades fisionômicas de Mata Atlântica no Rio de Janeiro. Tese de Doutorado. Universidade de São Paulo, São Paulo, 231p.

; Pessoa, S. V. A. \& Kurtz, B. C. 1997. Florística e estrutura do componente arbustivo-arbóreo de um trecho preservado de floresta montana na Reserva Ecológica de Macaé de Cima. In: Lima, H. C. \& Guedes-Bruni, R. R. (eds.). Serra de Macaé de Cima: diversidade florística e conservação em Mata Atlântica Jardim Botânico do Rio de Janeiro, Rio de Janeiro. Pp. 127-146.

Hoehne, F. C. 1941. Leguminosas Papilionadas (Machaerium e Paramachaerium). Flora Brasílica 25(3): 1-99.

Holmgren, P. K.; Holmgren, N. H. \& Barnett, L. C. (eds.). 1990. Index Herbariorum of the world. $8^{\text {th }}$ ed. New York Botanic Garden, New York, 693p.

Janzen, D. H. 1997. Florestas tropicais secas: o mais ameaçado dos grandes ecossistemas tropicais. In: Wilson,E.O. (org.). Biodiversidade. Nova Fronteira, Rio de Janeiro. Pp. 166-176.

Joly, C. A.; Aidar, M. P. M.; Klink, C. A.; McGrath, D. G.; Moreira, A. G.; Moutinho, P.; Nepstad, D. C.; Oliveira, A. A.; Pott, A.; Rodal, M. J. N. \& Sampaio, E. V. S. B. 1999. Evolution of the Brazilian phytogeography classification systems: implications for biodiversity conservation. Ciência e Cultura 51(5/6): 331-348.

Klitgaard, B. B. 2005. Platymiscium (Leguminosae: Dalbergieae): biogeography, systematics, morphology, taxonomy and uses. Kew Bulletin 60: 321-400.

Kurtz, B. C. \& Araujo, D. S. D. 2000. Composição florística e estrutura do componente arbóreo de um trecho de Mata Atlântica na Estação Ecológica Estadual do Paraíso, Cachoeiras de Macacu, Rio de Janeiro, Brasil. Rodriguésia 51(78/79): 69-111. 
Leitão-Filho, H. F. 1982. Aspectos taxonômicos das florestas do estado de São Paulo. Sivicultura em São Paulo 16a(1): 197-206.

Lewis, G. P. 1987. Legumes of Bahia. Royal Botanic Gardens, Kew, 369p.

Lewis, G. P.; Schrire, B.; Mackinder, B. \& Lock, M. (eds.). 2005. Legumes of the world. Royal Botanic Gardens, Kew, 577p.

Lima, H. C. 1983. Novos taxa de LeguminosaePapilionoideae (Tribo Dalbergieae) do Brasil. Bradea (3) 45: 399-405.

.1995. Leguminosas da Flora Fluminensis - J. M. C. Vellozo - Lista atualizada das espécies arbóreas. Acta Botânica Brasilica 9(1): 123-146.

2000. Leguminosas arbóreas da Mata Atlântica: uma análise da riqueza, padrões de distribuição geográfica e similaridades florísticas em remanescentes florestais do estado do Rio de Janeiro. Tese de Doutorado. Universidade Federal do Rio de Janeiro, Rio de Janeiro, 151p.

; Correa, C. M. B. \& Farias, D. S. 1994. Leguminosae. In: Lima, M. P. M. \& Guedes-Bruni, R. R. (orgs.). ReservaEcológica de Macaé de Cima - Nova Friburgo-RJ: aspectos floristicos das espécies vasculares. Vol. 1. Jardim Botânico do Rio de Janeiro, Rio de Janeiro. Pp. 167-228.

\& Guedes-Bruni, R. 1997. Plantas arbóreas da Reserva Ecológica de Macaé de Cima. In: Lima, H. C. \& Guedes-Bruni, R. R. (eds.). Serra de Macaé de Cima: diversidade florística e conservação em Mata Atlântica. Jardim Botânico do Rio de Janeiro, Rio de Janeiro. Pp. 53-64.

; Guedes-Bruni, R. R.; Sylvestre, L. S. \& Pessoa, S. V. A. 1997. Padrões de distribuição geográfica de espécies vasculares da reserva Ecológica de Macaé de Cima. In: Lima, H. C. \& Guedes-Bruni, R. R. (eds.). Serra de Macaé de Cima: diversidade florística e conservação em Mata Atlântica. Jardim Botânico do Rio de Janeiro, Rio de Janeiro. Pp. 103-123.
Maioli-Azevedo, V. 2008. Recursos vegetais utilizados pelos quilombolas de Machadinha, Quissamã, RJ. Dissertação de Mestrado. Museu Nacional do Rio de Janeiro, Rio de Janeiro, 119p.

Mansano, V. F. 1997. Estudos taxonômicos da tribo Swartzieae (DC.) Benth. (Leguminosae: Papilionoideae) no Sudeste do Brasil. Dissertação de Mestrado. Universidade Estadual de Campinas, São Paulo, 140p. \& Azevedo-Tozzi, A. M. G. 1999. Distribuição geográfica, ambiente preferencial e centros de diversidade dos membros da tribo Swartzieae na Região Sudeste do Brasil. Revista Brasileira de Botânica. 22 (2): 249-257.

Marques, M. C. M. 1997. Mapeamento da cobertura vegetal e listagem das espécies ocorrentes na Área de Proteção Ambiental de Cairuçu, Município de Parati, RJ. Jardim Botânico do Rio de Janeiro, Rio de Janeiro, 96p.

Mckey, D. 1994. Legume and nitrogen: The evolutionary ecology of a nitrogendemanding lifestyle. In: Sprent, J. I. \& Mckey, D. (eds.). Advances in Legume Systematics 5. The Nitrogen Factor. Royal Botanic Gardens, Kew, p. 211-228.

Mendonça Filho, C. V. 1996. Braúna, angico, jacarandá e outras leguminosas de mata atlântica: Estação Biológica de Caratinga, Minas Gerais. Belo Horizonte, Fundação Botânica Margaret Mee/Fundação Biodiversitas/AP.EBC/IEF/FZB-BH/SB. 2002. Citotaxonomia de Machaerium Pers. e revisão de Machaerium sect. Oblonga (Benth.) Taub. (Leguminosae - Papilionoideae). Tese de Doutorado. Universidade Estadual de Campinas, Campinas, 207p.

Mittermeier, R. A.; Myers, N. \& Thomsen, J. B. 1998. Biodiversity hotspots and major tropical wilderness areas: approaches to setting conservation priorities. Conservation Biology 12(3): 516-520.

Mooney, H. A.; Bullock, S. H. \& Medina, E. 1995. Introduction. In: Bullock, S. H.; 
Mooney, H. A. \& Medina, E. (eds.). Seasonally dry tropical forest. University Press, Cambridge. Pp. 9-34.

Mori, S. A.; Boom, B. M. \& Prance, G. T. 1981. Distribution patterns and conservation of eastern Brazilian coastal forest tree species. Brittonia 33: 233-245

1990. Diversificação e conservação das Lecythidaceae neotropicais. Acta Botânica Brasilica 4(1): 45-69.

Morim, M. P. 2006. Leguminosae arbustivas e arbóreas da Floresta Atlântica do Parque Nacional do Itatiaia, Sudeste do Brasil: padrões de distribuição. Rodriguésia 57(1): 27-45.

\& Barroso, G. M. 2007. Leguminosae arbustivas e arbóreas da Floresta Atlântica do Parque Nacional do Itatiaia, sudeste do Brasil: subfamílias Caesalpinioideae e Mimosoideae. Rodriguesia 58(2): 423-468.

Myers, N.; Mittermeier, R. A.; Mittermeier, C. G.; Fonseca, G. A. B. \& Kent, J. 2000. Biodiversity hotspots for conservation priorities. Nature 403: 853-858.

Nascimento, M. T. N. \& Lima, H. C. 2008. Floristic and structural relationships of a Tabuleiro forest in Northeastern Rio de Janeiro, Brazil. In: Thomas, W. W. (ed.). The Atlantic Coastal Forest of Northeastern Brazil. The New York Botanical Garden, New York. Pp.395-416.

Oliveira Filho, A.T. \& Ratter, J.A. 1995. A study of origin of Central Brazilian forest by the analysis of plant species distribution patterns. Edinburgh Journal of Botany 52(2): 141-194.

\& Fontes, M. A. L. 2000. Patterns of floristic differentiation among atlantic forests in southeastern Brazil, and the influence of climate. Biotropica 32(4b): 793-810.

Peixoto, A. L. \& Gentry, A. H. 1990. Diversidade e composição florística da mata de tabuleiro na Reserva Florestal de Linhares (Espírito Santo, Brasil). Revista Brasileira de Botânica 13: 19-25.

Pennington, T. D. 1997. The genus Inga: Botany. Royal Botanic Gardens, Kew, 844p.
Pennington, R. T.; Lavin, M.; Prado, D. E.; Pendry, C. A.; Pell, S. K. \& Butterwurth, C.A. 2004. Historical climate change and speciation: neotropical seasonally dry forest plants show patterns of both Tertiary and Quaternary diversification. Philosophical Transactions of the Royal Society of London (B) 359: 381-407.

; Lewis, G. P. \& Ratter, J. A. 2006. An overview of the plant diversity, biogeography and conservation of Neotropical Savannas and Seasonally Dry Forests. In: Pennington, R. T. \& Ratter, J. A. (eds.). Neotropical savannas and seasonally dry forests: diversity, biogeography, and conservation. Systematics Association Special Volumes, n. 69.

Pirani, J. R. 1990. Diversidade taxonômica e padrões de distribuição geográfica em Picramnia (Simaroubaceae) no Brasil. Acta Botanica Brasilica 4(1): 19- 44.

Prado, D. E. 1991. A critical evaluation of the floristic links between chaco and caating vegetation in South America. $\mathrm{PhD}$ thesis. University of Saint Andrews, Saint Andrews. \& Gibbs, P. E. 1993. Patterns of species distribution in the dry seasonal forest of South America. Annals of Missouri Botanical Gardens 80: 902-927.

Prance, G. T. 1979. The taxonomic and phytogeography of Chrysobalanaceae of the atlantic coastal forest of Brazil. Revista Brasileira de Botânica 2(1): 19-39.

Queiroz, L. P. 2006. The Brazilian caatinga: phytogeographical patterns inferred from distribution data of the Leguminosae. In: Pennington, R. T. \& Ratter, J. A. (eds.). Neotropical savannas and seasonally dry forests: diversity, biogeography, and conservation. Systematics Association Special Volumes, n. 69.

Ribeiro, R. D. \& Lima, H. C. 2007. Taxonomia de Machaerium incorruptibile (Vell.) Benth. e espécies afins (LeguminosaePapilionoideae) na Mata Atlântica. Rodriguésia 58(1): 17-25.

Richardson, J. E.; Pennington, T. D. \& Hollingsworth, P. M. 2001. Rapid 
diversification of a species-rich genus of Neotropical Rain Forest trees. Science 293 (5538): 2242-2245.

Rudd, V. E. 1987. Studies in Machaerium (Leguminosae) - V.Phytologia 62(4): 277-302.

Sá, C. F. C. 2006. Estrutura, diversidade e conservação de Angiospermas no Centro de Diversidade de Cabo Frio, estado do Rio de Janeiro. Tese de Doutorado. Universidade Federal do Rio de Janeiro, Rio de Janeiro, 250p.

Silva, G. C. \& Nascimento, M. T. N. 2001. Fitossociologia em um remanescente de mata sobre tabuleiros no norte do estado do Rio de Janeiro (Mata do Carvão). Revista Brasileira de Botânica 24(1): 51-62.

Spolidoro, M. L. C. V. 2001. Composição e estrutura de um trecho de floresta no médio Paraíba do Sul, RJ. Dissertação de Mestrado. Universidade Federal Rural do Rio de Janeiro, Seropédica, 100p.
Sprent, J.I. 1994. Nitrogen acquisition systems in the Leguminosae. In: Sprent, J.I. \& Mckey, D. (eds.). Advances in legume systematics 5 . The nitrogen factor. Royal Botanic Gardens, Kew. Pp. 1-16.

Ururahy, J. C.; Collares, J. E. R.; Santos, M. M. \& Barreto, R. A. A. 1983. Vegetação. In: RADAMBRASIL. Folhas SF 23/24 Rio de Janeiro/Vitório. Levantamento dos Recursos Naturais.

Veloso, H. P.; Rangel-Filho, A. L. R. \& Lima, J. C. A. 1991. Classificação da vegetação brasileira adaptada a um sistema universal. IBGE, Rio de Janeiro, 124p.

Warwick, M. C.; Lewis, G. P. \& Lima, H. C. 2008. A reappraisal of Barnebydendron (Leguminosae: Caesalpinioideae: Detarieae). Kew Bulletin 63: 143-149.

WWF \& IUCN. 1997. Centres of plant diversity. A guide and strategy for their conservation. Vol. 3. IUCN Publ. Unit. Cambridge. 